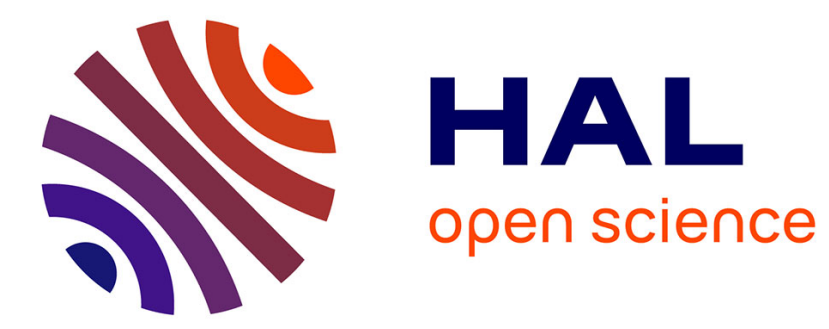

\title{
Autisme et addictions
}

Chantal Lheureux-Davidse

\section{To cite this version:}

Chantal Lheureux-Davidse. Autisme et addictions. Recherches en psychanalyse, 2005, L'informe et l'archaïque, 1 (3), pp.31 - 42. 10.3917/rep.003.0031 . hal-01513877

\section{HAL Id: hal-01513877 https://hal.science/hal-01513877}

Submitted on 25 Apr 2017

HAL is a multi-disciplinary open access archive for the deposit and dissemination of scientific research documents, whether they are published or not. The documents may come from teaching and research institutions in France or abroad, or from public or private research centers.
L'archive ouverte pluridisciplinaire HAL, est destinée au dépôt et à la diffusion de documents scientifiques de niveau recherche, publiés ou non, émanant des établissements d'enseignement et de recherche français ou étrangers, des laboratoires publics ou privés. 


\section{AUTISME ET ADDICTIONS}

Chantal Lheureux-Davidse

Association Recherches en psychanalyse | «echerches en psychanalyse »

2005/1 n 3 | pages 31 à 42

ISSN 1767-5448

ISBN 2847950486

Article disponible en ligne à l'adresse :

http://www.cairn.info/revue-recherches-en-psychanalyse-2005-1-page-31.htm

\section{Pour citer cet article :}

Chantal Lheureux-Davidse, "Autisme et addictions », Recherches en psychanalyse 2005/1 (n 3 ), p. 31-42.

DOI 10.3917/rep.003.0031

Distribution électronique Cairn.info pour Association Recherches en psychanalyse.

(C) Association Recherches en psychanalyse. Tous droits réservés pour tous pays.

La reproduction ou représentation de cet article, notamment par photocopie, n'est autorisée que dans les limites des conditions générales d'utilisation du site ou, le cas échéant, des conditions générales de la licence souscrite par votre établissement. Toute autre reproduction ou représentation, en tout ou partie, sous quelque forme et de quelque manière que ce soit, est interdite sauf accord préalable et écrit de l'éditeur, en dehors des cas prévus par la législation en vigueur en France. Il est précisé que son stockage dans une base de données est également interdit. 


\title{
Autisme et addictions
}

\author{
Chantal Lheureux-Davidse
}

Les questions de la forme et de l'informe se posent dans les problématiques addictives comme dans l'autisme. Quels liens et quelles différences peut-on repérer entre l'autisme et les addictions quant à ces questions? A partir de cas cliniques issus de ces deux champs, je propose de mettre à l'épreuve l'hypothèse de l'existence d'un noyau autistique chez certaines personnes souffrant d'addiction alimentaire ou d'addiction à des sensations fortes. Je parlerai de la perte du sentiment d'exister et de ses conséquences dans l'autisme et les pathologies addictives. Puis je dégagerai la spécificité des zones corporelles effacées lors du démantèlement sensoriel dans les pathologies autistiques, boulimiques et anorexiques, en m'appuyant sur les étapes de construction de l'image du corps. Je préciserai les destins différents de la dispersion psychique lors des moments de clivage.

Enfin, je développerai la question de l'urgence du recours aux sensations fortes chez les patients souffrant d'angoisses catastrophiques.

\section{LA PERTE DU SENTIMENT D'EXISTER DANS SON CORPS}

Les compulsions addictives obéissent souvent à un processus qui tente de maintenir un sentiment d'existence malgré un attrait pour une dispersion psychique pouvant entraîner des effets de démantèlement sensoriel. Le « démantèlement sensoriel » a été essentiellement mis en évidence par Donald Meltzer à partir de cas d'autisme ${ }^{1}$. Cette expression désigne la perte progressive de la conscience des sensations cor-

1. Meltzer D., Bremner J., Hoxter S., Wittenberg I., Explorations in Autism, trad. Fr. Explorations dans le monde de l'autisme, The Roland Harris Educational Trust, 1980, p.266. 
porelles qui sont normalement en lien entre elles dans ce qu'il appelle une consensualité. Il se produit alors une perte du «moi corporel » dans le sens Freudien et l'annulation progressive du « sentiment d'exister en continu» dans le sens Winnicottien ${ }^{2}$.

Freud écrit en 1923 dans «Le moi et le ça»:

«Le moi est avant tout un moi corporel, il n'est pas seulement un être de surface, mais lui-même la projection d'une surface. » Dans la traduction anglaise de 1927, on ajoute avec l'autorisation de Freud : «C'est-à-dire : le moi est finalement dérivé de sensations corporelles, principalement de celles qui ont leur source dans la surface du corps. Il peut ainsi être considéré comme une projection mentale de la surface du corps... $»^{3}$

Pourquoi la question de la conscience des sensations corporelles est-elle si essentielle pour se sentir exister?

Lors de clivages entre la psyché et le soma, la perte de la conscience des sensations éprouvées par le corps empêche la personne de se sentir concernée par ellemême et bloque toute communication interpersonnelle.

Le phénomène entraîne un mode de pensée essentiellement associatif qui s'éloigne peu à peu de toute pensée logique de cause à effet. C'est-à-dire que la pensée fonctionne alors par effet de résonance, hors contexte de toute temporalité historique.

Cette pensée essentiellement associative, si elle n'est plus reliée avec souplesse à une pensée logique contextualisée, peut dériver peu à peu jusqu'à la perte de tout lien avec le moi corporel. Les pensées tournent en rond et sont accélérées dans leur mouvement jusqu'à des effets de saturation ou de vertiges. La liaison psyché/soma se trouve en quelque sorte coupée. Les pensées ne peuvent plus s'apaiser. L'image du corps se défait progressivement jusqu'à ce que des paniques liées au fait de ne plus se sentir exister dans son corps déclenchent des angoisses insoutenables d'anéantissement ${ }^{4}$.

La perte de contact avec les ressentis corporels, associée à la perte d'une capacité à organiser la pensée dans une logique de cause à effet, bloque toute capacité à initier un mouvement. Parfois, la motricité spontanée ne peut plus s'exercer. Seule l'action des pensées peut provoquer un mouvement, mais d'une façon mécanique, sans que la personne se sente concernée pour autant dans son corps. Dans d'autres cas, la motricité volontaire elle-même peut devenir impossible à mettre en œuvre.

La perte de la conscience des ressentis corporels empêche de se sentir concerné par soi-même. Tout choix personnel devient alors impossible. La communication avec autrui perd aussi toute spontanéité. La réponse à une question posée se fait alors

2. Winnicott D., 1971, Creativity and its Origins, trad. Cl. Monod et J.-B. Pontalis, «La créativité et ses origines », in Jeu et réalité, Paris : Gallimard, 1975.

3. Freud S., 1923 (fin 1922), Das Ich und das Es, Le moi et le ça, in (1915-1922), Essais de psychanalyse, trad. coll., P.B.P Payot, Paris 1918, p. 238, (1921-1923), Sigmund Freud, Euvres complètes, vol. XVI, trad. coll., P.U.F., Paris, 1991, p. 270.

4. Winnicott D., 1963, The fear of Breakdown, trad. J. Kalmanovitch et M. Gribinski, «La crainte de l'effondrement », in Figures du vide, Nouvelle Revue de Psychanalyse, Paris : Gallimard, nrf, 1975, 11, pp. 35-44. 
soit de façon automatique comme dans une situation connue, soit elle est donnée en différé, le temps de se sentir concerné. Souvent la réponse en différé aurait pu être tout à fait adéquate si elle avait pu être énoncée spontanément au bon moment. L'impression de vivre en décalage du corps est souvent décrite. Les commandes motrices ne peuvent plus répondre aux souhaits organisés par la pensée, particulièrement pour commencer un mouvement qui a perdu sa logique d'enchaînement de cause à effet.

\section{LES ZONES CORPORELLES EFFACÉES LORS DU DÉMANTÈLEMENT SENSORIEL}

Existe-t-il une spécificité des zones corporelles effacées lors du démantèlement sensoriel, selon les organisations psychopathologiques? L'étude des cas cliniques, dans leur singularité, nous éclaire sur ce sujet.

Si l'angoisse de vide intérieur et la crainte de dispersion en dehors de son corps sont souvent présentes durant des crises de boulimie, c'est plutôt l'angoisse de déformation et d'envahissement dans le corps qui est prégnante dans les discours des personnes souffrant d'anorexie.

Dans l'autisme, ce sont aussi des angoisses d'arrachement de différentes parties du corps qui sont à l'œuvre, par exemple l'arrachement des extrémités du corps et de la zone buccale.

Des terreurs de perte du sentiment d'exister sont liées à l'effacement provisoire de la conscience des ressentis corporels au fur et à mesure que se déconstruit l'image du corps. Le refuge dans les pensées et l'adhésivité aux mouvements de ses pensées font perdre le contact avec la conscience des ressentis corporels, dans des moments de clivage entre la psyché et le soma. Ce n'est pas la représentation du corps qui est en danger d'effacement mais la conscience des ressentis corporels.

Que ce soit dans l'autisme, dans la boulimie ou dans l'anorexie, il existe différents degrés d'effacement de l'image du corps selon l'ampleur du démantèlement. Lorsque l'effacement de certaines parties du corps se prolonge, il peut s'étendre à d'autres zones jusqu'à ce que des angoisses d' anéantissement surviennent et déclenchent une recherche dans l'urgence de la réversibilité du processus. Les parties concernées peuvent être variables selon chaque cas et parfois communes aux trois pathologies. Les dernières parties du corps intégrées dans l'image du corps seront les premières défaites lors du processus de démantèlement sensoriel.

L'inconcevabilité d'une situation traumatique non intégrable pour la psyché peut entraîner une déconstruction de l'image du corps jusqu'à ses origines.

Le démantèlement sensoriel associé à la perte de liens entre les sens peut alterner avec un remantèlement au cours duquel l'image du corps se reconstruit aux endroits où elle avait été effacée de la conscience. Ce processus réversible est normal tant qu'il s'opère avec souplesse et tant qu'il n'entraîne pas un clivage destructeur entre psyché et soma.

Dans les cas plus pathologiques, comme dans la boulimie grave ou dans l'autisme, le remantèlement sensoriel ne se fait souvent qu'au prix de la douleur ou de sensa- 
tions fortes. Cette douleur est recherchée de façon compulsive à la suite d'angoisses d'anéantissement liées à la perte du sentiment d'exister dans son corps ${ }^{5}$.

La réanimation sensorielle peut concerner toute zone corporelle effacée de la perception. L'étude du processus de formation de l'image du corps nous éclaire sur ce sujet.

\section{L'IMAGE DU CORPS CHEZ LES ENFANTS AUTISTES}

Geneviève Haag a bien décrit les étapes de construction de l'image du corps à partir de la clinique de l'autisme ${ }^{6}$. J'en rappelle les principales étapes: le sentiment d'existence d'un « tout autour » précède le sentiment d'exister à l'intérieur. De même, l'investissement de la tête précède l'investissement du reste du corps.

Quant à l'investissement du regard, il se construit chez le bébé lors de l'accordage avec le regard maternel lorsque celui-ci est bien vivant et présent, c'est-à-dire qu'il manifeste les qualités de brillance et de mouvement. Durant cet échange que le bébé recherche tout autant que la mère, le bébé est porté dans les bras de sa mère qui soutient son dos et sa nuque avec douceur, fermeté et chaleur.

Ainsi la constitution du dos, de cet arrière-plan vertical de sécurité, se fait conjointement à l'installation du regard. Comme si le dos devenait le fond des yeux et constituait un contenant de sécurité pendant l'investissement du lien à l'autre par le regard. C'est dans ces conditions que la tridimensionnalité peut s'installer. Le corps devient capable de contenir de bonnes expériences et de les intérioriser.

Quand un enfant autiste regarde très près les yeux de l'autre, il recherche sans doute s'il s'y trouve un fond solide, doux et contenant, et il va souvent inspecter conjointement la nuque, l'arrière-plan de la tête ou le haut du dos pour vérifier s'il est troué ou bien fermé. Ainsi il vérifie, en se lançant dans le regard de l'autre, s'il est contenu ou s'il risque d'être absorbé dans un trou noir sans fond comme a pu le décrire Francès Tustin ${ }^{7}$.

Quand le regard s'installe, l'axe vertical du dos fait charnière entre les hémicorps droite et gauche, d'abord au niveau de la tête puis dans tout le corps. Puis l'investissement de la charnière horizontale « accrochera » la tête au bas du corps. Des mouvements d'articulation entre le haut et le bas du corps en témoignent. Ensuite les extrémités du corps, mains et pieds, sont accrochées au reste de l'image du corps déjà constituée. Puis l'investissement de la peau contenant l'ensemble du corps permettra que les orifices soient ensuite sphinctérisés psychiquement.

5. Barabe N., Lheureux-Davidse C., «La question de la réversibilité du démantèlement chez un adulte autiste », in Cliniques méditerranéennes, $n^{\circ} 70$, éditions Erès, 2004.

6. Haag G., Aux sources de la vie; le langage préverbal et l'émergence des représentations du corps en situation psychanalytique individuelle ou groupale avec des enfants autistes, «Actes des langage. Quand parler c'est agir», Dialogue, n²3, Paris, 1er trimestre 1994, pp. 40-58.

7. Tustin F., 1986, Autistic Barriers in Neurotic Patients, Londres : Karnac Books, trad. Paul Chemla, Le trou noir de la psyché, Barrières autistiques chez les névrosés, Paris: Seuil, 1989. 
Une image du corps constituée n'est plus une somme de parties accrochées les unes aux autres mais devient intègre grâce à une consensualité. C'est-à-dire que chaque partie est en lien avec le tout. Les sens sont alors reliés entre eux, tout en étant différenciés.

Lorsque l'image du corps est constituée dans cette consensualité, le sentiment d'exister permet également de se sentir relié aux autres par des communications. Tant que cette consensualité n'est pas mise en place, comme dans une mise en réseau, l'investissement d'un sens ne peut se faire que d'une façon exclusive sans pouvoir se relier à un autre sens. De même l'investissement d'une personne efface les autres. La permanence de l'objet se trouve dès lors difficile à intégrer, chaque changement représentant une menace d'anéantissement.

La zone buccale, pendant l'investissement du stade oral, constitue l'une des zones les plus fragiles de l'image du corps. Lorsque cette partie n'est pas investie, cela provoque une vulnérabilité de tout le corps accompagnée d'angoisses d'intrusion ou de vidage.

Par ailleurs, certains enfants présentant des composantes autistiques sont assaillis par l'angoisse de se vider entièrement si leur corps est ouvert, par exemple si une petite blessure de leur peau n'est pas encore refermée, ou encore au moment des toilettes lorsqu'une partie de leur contenu part de leur corps. Ils peuvent alors s'identifier à ce contenu digestif qu'ils abandonnent au point d'en oublier le reste de leur corps. Il n'est pas rare que l'enfant pris de panique lutte contre ses angoisses en tentant de boucher l'orifice des toilettes ou en vérifiant de façon compulsive qu'il y a toujours de l'eau en réserve dans la chasse d'eau ou dans les robinets. Ne pouvant plus s'identifier à lui-même, il s'identifie à tout ce qui fait écho dans son environnement aux parties de son image du corps à reconstituer ${ }^{8}$. Toute ouverture perçue dans l'environnement devient la métaphore des orifices corporels.

C'est sans doute ainsi que l'on peut comprendre les compulsions des enfants autistes à ouvrir et fermer les portes ou les volets. Ils vérifient que les orifices de leur corps ou leurs yeux peuvent être ouverts ou fermés. Dans ce cas, le dedans est alors limité et différencié du dehors, il demeure en sécurité sans annuler le dehors durant les compulsions.

Lors des processus de remantèlement sensoriel, les personnes autistes ont souvent recours à des stéréotypies visant à obtenir, par des effets visuels, des formes lumineuses géométriques en léger mouvement, par exemple en agitant leurs doigts devant les yeux en direction d'une source lumineuse. Ils obtiennent alors les qualités de brillance accompagnées de légers mouvements que les enfants normaux trouveraient dans les échanges de regard.

Leur fascination pour les formes géométriques colorées en léger mouvement n'est-elle pas une préparation à se sentir contenu dans une forme identifiante bien vivante, malgré qu'elle soit encore abstraite?

8. Lheureux-Davidse C., L'autisme infantile ou le bruit de la rencontre, Contribution à une clinique des processus thérapeutiques, Paris: L'Harmattan, 2003, 342 p. 
Adam, un jeune autiste s'exprimant dans un langage très métaphorique, m'expliquait qu'il avait le sentiment d'être un carré lorsqu'il retrouvait ses sensations corporelles. Il décrivait la façon dont il réintégrait son corps par le haut de la tête en arrière-plan et le fait que son dos ouvert se refermait. Puis il précisait que son corps était comme «une piscine bien fermée 24 h sur 24 avec insistance». Enfin il s'émerveillait du monde qu'il semblait découvrir dans sa beauté et parlait d'une «vitrine avec des trésors immenses à l'intérieur». Il utilisait le chiffre 4 et les formes carrées pour témoigner de son sentiment d'être contenu dans des formes identifiables et définies. Il pouvait alors laisser surgir ses émotions.

Certains enfants autistes peuvent se réfugier dans des recherches sensorielles rassurantes qu'ils ont vécues avant les moments catastrophiques. Ainsi les balancements rythmiques ne font-ils pas écho aux rythmes cardiaques et respiratoires rassurants par leur continuité, que le fœtus percevait de sa mère in utero?

\section{LES ANGOISSES DE VIDE INTÉRIEUR ET DE PERTE DES LIMITES DANS LA BOULIMIE}

Dans le cas de la boulimie, des états de dispersion psychique peuvent aller jusqu'à la perte de contact avec les ressentis corporels propres. Le vécu décrit est celui d'un vide intérieur, entre autres causé par l'angoisse de perte de la conscience des sensations internes et par une précarité du sentiment d'être contenu dans son corps par une enveloppe ou un environnement solide. L'existence d'un intérieur est mise en doute jusqu'à susciter un risque d'anéantissement. Ces angoisses mettent bien en péril le sentiment d'existence.

Chez ces patients, l'effacement d'un dedans génère une perte de la notion de profondeur qui se trouve à l'origine d'angoisses spatiales. Le sujet est plongé dans un état de vulnérabilité qui fait écho à la période de mise en place de la tridimensionnalité. Le corps est vécu comme un feuillet à deux dimensions qui ne pourrait avoir que des contacts de surface, comme avant l'installation du regard. Remarquons plus généralement que c'est la mise en place du regard qui fonde toute la profondeur de la relation à l'autre et qui donne accès à la tridimensionnalité. Même si le bébé se prépare déjà à la notion de profondeur avec un autre sens, celui du toucher, lorsqu'il teste l'élasticité de la peau de sa mère en exerçant de légères pressions sur son sein lors de la tétée par exemple, ou lorsqu'il explore les aspérités des surfaces.

Les moments de vide intérieur éprouvés par de nombreux patients boulimiques traduisent des difficultés d'intériorisation de bonnes expériences relationnelles répétées. Ces phénomènes entraînent une difficulté à mémoriser ce qui a été vécu pour éviter d'être en contact avec des souvenirs d'expériences douloureuses. Cette problématique fait écho aux états dépressifs chez l'enfant, lorsque ses pensées sont tournées vers ses parents pour qui il s'inquiète au point d'oublier sa propre existence et ses propres ressentis. L'enfant n'arrive pas à se sentir concerné par lui-même lorsqu'il apprend. Dès lors, il oublie aussitôt lorsqu'il apprend et il en perd toute capacité d'intériorisation de ses bonnes expériences. 
Les paniques de perte du sentiment d'exister se déclenchent parfois au moment où la conscience de sentir ses limites corporelles est également mise en péril. Les angoisses d'effacement d'un dedans menacent l'existence du contenant. C'est bien en éprouvant la solidité de la contenance par des compulsions de remplissage que la réassurance d'un sentiment d'exister dans ses propres bords peut avoir lieu.

Voici la description qu'une jeune femme ayant souffert de boulimie faisait d'ellemême :

«Lorsque je change d'endroit, je suis angoissée. J'ai l'impression d'être réduite à un tube creux placé verticalement qui ne serait fermé ni en haut ni en bas, vide à l'intérieur. La densité de l'air est tellement légère que je ne me sens en sécurité ni dedans ni dehors. Cela me fait penser que jusqu'à 6 ans je n'étais pas propre la nuit. Je me sentais complètement seule particulièrement lorsque j'étais en vacances avec mes grands-parents. Ils m'ont gardée jusqu'à mes 6 ans toute l'année et pendant les vacances. J'avais l'impression d'être dans une profonde solitude. Ma mère $m$ 'a récupérée à 6 ans. Elle critiquait ma grand-mère de m'avoir donné trop à manger. Al'adolescence je devais monter chaque jour sur la balance sous les regards de mes parents situés derrière mes épaules. C'était épouvantable.

A d'autres moments au contraire, je sens qu'il y a une pression énorme à l'intérieur de mon corps, comme si la densité était très forte. J'ai l'impression que mon enveloppe est prête à se craqueler, comme dans un film de science fiction où il y aurait plein de lumière à l'intérieur qui s'échapperait à travers les craquelures. »

C'est dans ces moments d' angoisse de risque d'anéantissement que les crises de boulimie se déclenchent.

Dans certains cas de boulimie avec des noyaux autistiques, les compulsions de remplissage s'actualisent pour tenter de colmater la béance d'une zone buccale non constituée. Dans d'autres cas de boulimie, ces compulsions de remplissage peuvent être dues à des angoisses de vide intérieur. Il s'agit là plutôt de difficulté d'intériorisation par perte d'investissement d'un «dedans ».

Dans de nombreux cas de boulimie, le poids réel du corps fait office de mémoire à penser d'un lourd traumatisme, comme celui d'abus sexuel ou de vécu incestuel.

\section{LE PARADOXE DU TROP PLEIN DANS L'ANOREXIE}

Dans l'anorexie, l'appréhension de l'image du corps est souvent envahie par des représentations incestuelles impensables ${ }^{9}$. Pour effacer un envahissement psychique et un vécu intérieur de sensations d'obésité, sont organisées des phobies d'ingestion de certaines nourritures qui deviennent interdites et inavalables dans le réel.

Il s'agirait d'un corps habité par des imagos parentales trop souffrantes, dont l'enfant serait le dépositaire et le gardien. Dans des représentations impensables qui cher-

9. Racamier P.-C., Le génie des origines. Psychanalyse et psychoses, Paris : Payot, collection Bibliothèque scientifique, 1992, $420 \mathrm{p}$. 
cheraient à se manifester sous une forme purement hallucinatoire sensoriellement, comme des focalisations sur une image réelle de toute façon trop grosse, il faudrait perdre dans le réel le poids psychique impensable inassimilable.

Dans l'anorexie, le vécu corporel peut être décrit comme un ensemble de sensations de déformation pouvant aller jusqu'à la dysmorphophobie. Il s'agit souvent d'un sentiment d'être habité par une forme à laquelle il serait dangereux de s'assimiler, qui déforme jusqu'à l'informe ${ }^{10}$ et dont il faudrait se débarrasser à tout prix.

Une jeune fille présentant des tendances anorexiques relatait au cours de son travail analytique le rêve suivant:

« Je me regarde dans la glace et au lieu de me voir, je vois le corps de ma mère à la place du mien. J'étais tellement effrayée que je me suis réveillée en hurlant ».

Elle guettait devant la glace la moindre ride qu'elle pourrait apercevoir et avait l'impression d'avoir un bassin très large. Elle se sentait énorme à l'intérieur et en cherchait la preuve dans la glace, elle était terrorisée à l'idée que son corps montrerait de la cellulite qui déformerait sa silhouette. Elle vivait son corps comme une somme de parties qui n'étaient pas reliées entre elles.

«Parfois j'ai l'impression d'avoir un corps de vieille dame. Si je dépasse un certain poids, je me sens complètement en danger au gramme près. Quand je me vois énorme dans la glace, je me prive de manger le temps nécessaire pour retomber dans mes limites que je ne peux pas dépasser. C'est généralement le poids de la balance qui me l'indique.»

La preuve d'un petit poids à ne pas dépasser garantirait ainsi une barrière symbolique anti-incestuelle entre les générations et éviterait les angoisses de confusion des corps entre sa mère et elle-même. C'est ainsi que la patiente maintenait, en se privant de manger, une silhouette pré-pubère se différenciant nettement de celle de sa mère. Lorsqu'elle était petite fille, on lui reprochait d'être un peu trop ronde. Lors de l'entrée dans sa puberté qu'elle avait vécue comme trop précoce, elle était encombrée de formes qui poussaient, déformaient son corps et qui focalisaient de façon intrusive le regard des autres sur son intimité. Ses formes naturellement féminines se confondaient avec les rondeurs qui lui étaient reprochées dans la petite enfance. Elle n'était pas prête à se laisser modifier par des formes de femme qui modifiaient sa silhouette malgré elle.

Il apparût qu'elle avait dû remplacer auprès d'une mère fragile son père régulièrement absent. Elle recueillait les plaintes de sa mère qui lui confiait sa fragilité. Afin de protéger sa mère, elle s'effaçait provisoirement de sa place d'enfant pour essayer de contenir les angoisses de sa mère. En la prenant en charge trop souvent, elle se retrouvait prise dans une inversion de rôle générationnel au point de confondre le corps de sa mère et le sien. Néanmoins, elle ne supportait pas qu'on lui rappelle qu'elle était le portrait de sa mère. En se souvenant de ces moments elle me disait:

«Quand je me remémore cette période, j'ai l'impression que l'ambiance de la

10. Le Poulichet S., Psychanalyse de l'informe Dépersonnalisations, addictions, traumatismes, Paris : Flammarion, Aubier Psychanalyse, 2003, 125 p. 
maison était celle de l'intérieur d'un caveau fermé tout noir sans lumière. Ma mère était trop fragile. Elle n'a jamais su qui était son père ».

La question de la forme dans l'anorexie serait ainsi liée à l'évitement d'un envahissement par une forme hébergée à l'intérieur qui risquerait de déformer l'image du corps. Le souhait d'être réduite à une structure osseuse dépourvue de toute trace de féminité viserait à éviter, comme dans les cas graves d'anorexie, tout risque de confusion de génération qui serait un équivalent incestuel. La limite d'un poids impossible à dépasser participerait à la restauration d'une barrière psychique anti-incestuelle protectrice. La dysmorphophobie tenterait de réanimer, sur un mode hallucinatoire, une image du corps mortifère inanimée.

\section{LES DESTINS DE LA DISPERSION PSYCHIQUE}

Selon les pathologies autistiques ou addictives, les tendances à la dispersion psychique lors de clivages entre les pensées et les ressentis corporels ont des destins différents. Pendant les expériences de refuge dans les pensées, l'environnement est essentiellement vécu comme non humain ${ }^{11}$ et même parfois comme inanimé chez des personnes autistes qui se sont coupées de la relation à autrui, contrairement aux personnes souffrant d'addictions qui restent reliées à un environnement humain animé.

L'identification à un monde inanimé à réanimer ou à un environnement non humain à réhumaniser est d'autant plus forte chez les personnes souffrant d'autisme que leur sentiment d'exister ainsi que l'altérité sont encore très peu constitués en continu. Pendant les phases de clivage, des tentatives de reconstruction de leur image du corps et de la relation à l'autre les attireront dans des recherches de qualités ou de sensations trouvées dans l'environnement non humain et feront résonance avec des sensations ou des qualités normalement vécues lorsque l'image du corps est bien constituée et dans la relation à l'autre.

Par exemple les qualités de brillance associées aux micro-mouvements provoqués par leurs stéréotypies gestuelles, entre leurs yeux et le reflet d'une flaque d'eau, seront un équivalent métaphorique d'un échange de regard à réanimer. De même la qualité de verticalité recherchée dans des objets de leur environnement les prépare à une intégration de la qualité de verticalité de leur axe vertébral dans leur image du corps non encore constituée en continu.

Alors que chez des personnes souffrant d'addictions, la dispersion psychique lors de clivages entre la psyché et le soma restera plus facilement en lien avec un environnement humain. Par exemple l'identification aux personnes de l'entourage est parfois si forte qu'elle aboutit à l'effacement, à l'envahissement ou à la déformation du moi corporel selon les pathologies de boulimie ou d'anorexie. Le main-

11. Searles H., 1960, The nonhuman environment, trad. fr. D. Blanchard, L'environnement non humain, nrf, Gallimard, 1986, 398 p. 
tien de l'existence d'un entourage humain protecteur dépendrait d'une mise en suspens de leur sentiment d'exister au profit d'un narcissisme à reconstituer dans leur entourage.

\section{L'URGENCE DU RECOURS AUX SENSATIONS FORTES}

Lorsque la conscience des limites a été effacée ou perdue, il se produit un état d'indifférenciation et une perte sensorielle d'une forme identifiante. Et la perte de la conscience de l'espace interne provoque souvent des angoisses de perte des limites corporelles, à l'origine d'angoisses spatiales. Lorsqu'un trop long temps de perte de conscience des sensations corporelles s'est écoulé, le processus de démantèlement n'est souvent réversible qu'au prix de moyens sensoriels intenses. La fonction de ces derniers est de restaurer au plus vite la conscience des sensations corporelles perdues. Pour lutter contre des angoisses d'effacement, de liquéfaction, d'effondrement ou d'anéantissement, l'urgence de retrouver un sentiment d'exister dans son corps provoque la recherche précipitée de sensations fortes éprouvées dans le corps ${ }^{12}$.

Je pense que la douleur des automutilations ou celle provoquée par des compulsions addictives, ou encore le dépassement des limites, ont la même fonction réanimatrice de la conscience des sensations corporelles, même s'il ne s'agit pas des mêmes lieux à réanimer. Le passage des sensations inconscientes aux sensations conscientes et à la perception restaure le sentiment d'exister dans son corps.

Dans l'autisme, les auto-morsures des mains sont ainsi infligées avec force jusqu'à ce que les sensations réapparaissent à la conscience au prix de la douleur. Cette dernière restaure le sentiment d'exister jusque dans ses extrémités. Lorsque la charnière horizontale n'est pas constituée psychiquement, la perte de conscience du bas du corps peut engendrer des tentatives de réanimation sensorielle inconscientes. Des compulsions à se cogner les jambes ou les pieds ou à se tordre les chevilles restaurent une conscience sensorielle du bas du corps. Les auto-agrippements musculaires externes ou internes qui luttent contre les angoisses d'effondrement, provoquent des crampes douloureuses qui peuvent également occuper cette fonction réanimatrice de sensations. La recherche de douleur ou de sensations fortes ne représente pas un but en soi mais un moyen d'obtenir rapidement la restauration de sensations perdues ${ }^{13}$.

Parallèlement, dans une compulsion alimentaire, l'ingestion de tout un plat ou de tout un paquet de gâteaux semble nécessaire pour calmer l'angoisse. Dans la bou-

12. Lheureux C., «L'image du corps d'une personne abusée dans son enfance», in Détresse sociale et souffrance psychique, l’enjeu du sujet, Transhumances $n^{\circ} 4$, Presses Universitaires de Namur en Belgique, nov. 2003, pp. 195-198.

13. Lheureux-Davidse C., «La reconstruction de l'image du corps de Léo, enfant trisomique avec des retraits autistiques ", in Cliniques méditerranéennes, ${ }^{\circ} 69$, éditions Erès, mars 2004, pp. 291 310 . 
limie, le remplissage compulsif de nourriture en excès ne peut s'arrêter qu'à l'obtention d'un ressenti conscient de nausées, de maux au ventre ou de maux de tête. Le moindre reste oublié risquerait de raviver le sentiment d'être soi-même oublié et participe aux angoisses d'abandon. Cet effet de limite, de bord, permet de reprendre conscience d'un ressenti corporel qui rassure rapidement sur le sentiment d'exister sensoriellement dans un corps différencié de son environnement. Ainsi des limites contenantes sont à nouveau éprouvées.

Il me semble important de cesser de parler de la jouissance masochiste des personnes souffrant d'addictions. En effet lorsqu'elles utilisent la douleur comme moyen de réanimation sensorielle dans l'urgence pour retrouver un sentiment d'existence perdu, il me paraît inadéquat de parler de jouissance de la douleur. Par contre c'est dans le moment de retrouvaille avec un sentiment d'exister, à la suite d'angoisses innommables d'anéantissement, que peut apparaître, au prix parfois de la douleur, une jubilation de se sentir exister à nouveau dans son corps, ce qui n'a rien à voir avec une jouissance masochiste.

De même, les conduites sexuelles addictives recherchées dans l'urgence et souvent sans tendresse, ainsi que les sensations fortes procurées par des pratiques sado-masochistes, pourraient parfois être comprises comme des recherches de sensations corporelles réanimatrices, restaurant rapidement un sentiment d'exister dans son corps au cœur de moments d'angoisses catastrophiques. La prise de toxique pourrait également être interrogée sous cet angle.

Dans un cadre thérapeutique, les mouvements destructeurs engendrés par le recours à des sensations fortes réanimatrices peuvent être progressivement relayés par l'intensité partagée d'instants d'émerveillement. Les métaphores deviennent ainsi sources d'identification par leurs qualités qui éveillent par résonance celles d'une forme identifiante.

C'est dans ces moments me semble-t-il que la nomination des sensations par le thérapeute peut concourir au remantèlement sensoriel et favorise ainsi le passage des sensations inconscientes à la perception. L'interchangeabilité des sens cèdera la place à une différenciation retrouvée de chaque sens et sera le prélude à une consensualité restaurée. Ceci participe à la stabilité d'une image corporelle souple avec la psyché.

Le lien entre l'autisme et les addictions résiderait dans une difficulté à mettre en place une forme identifiante stable et le sentiment d'exister dans son corps. Le refuge dans les pensées clivées des ressentis corporels évite le contact avec les souvenirs de vécus catastrophiques. Mais la tendance à la dispersion psychique qui en découle peut déconstruire l'image du corps jusqu'à l'effacement.

Dans l'autisme, la dispersion psychique entraîne une identification à un environnement non humain inanimé à réhumaniser et à réanimer. Alors que dans les addictions, la dispersion psychique reste reliée à un environnement humain à renarcissiser.

Le démantèlement sensoriel provoque des angoisses d'anéantissement qui déclenchent une recherche dans l'urgence de la réversibilité du processus. Après un effacement prolongé de la conscience des ressentis corporels, le recours aux sensations fortes au prix de la douleur restaure provisoirement le sentiment d'exis- 
tence. Les zones corporelles concernées lors du démantèlement sensoriel réversible peuvent être communes à l'autisme et aux addictions. Le degré d'effacement de l'image du corps est à repérer dans la singularité de chaque cas.

Chantal LHEUREUX-DAVIDSE

Chargée d'enseignement Université Paris 7

59 rue du Temple

75004 Paris

\section{Chantal Lheureux-Davidse - Autisme et addictions}

Résumé : La mise en place d'une forme identifiante et du sentiment d'exister dans son corps reste problématique chez les personnes souffrant d'autisme ou d'addictions. Le refuge dans les pensées ou dans une dispersion psychique évite le contact avec des vécus catastrophiques, mais entraîne un processus de démantèlement sensoriel et une déconstruction de l'image du corps, dans des degrés divers selon chaque cas. L'effacement prolongé de la conscience des ressentis corporels provoque de telles angoisses d'anéantissement que le recours dans l'urgence aux sensations fortes au prix de la douleur restaure provisoirement le sentiment d'existence.

Mots-clés: Addictions - Autisme - Conscience des sensations corporelles Démantèlement - Douleur - Effacement - Pensées - Réversibilité - Sensations fortes Sentiment d'exister.

\section{Chantal Lheureux-Davidse - Autism and addictions}

Summary : Putting in place an identifying form and the feeling of existence in the body remains problematic with the persons suffering from autism or addictions. The refuge in thoughts or in psychic dispersion evades contact with the catastrophic experiences, but leads to a process of sensorial dismantling and a deconstruction of the bodily image, to a different degree in each case. The prolonged effacement of the consciousness of feeling the body provokes such angst of annihilation that a provisional return to the feeling to exist is sought by urgently creating strong sensations at the price of pain.

Key-words : Addictions - Autism - Conscience of bodily sensations - Dismantling Pain - Effacement - Thoughts - Reversibility - Strong sensations - Feeling of existence. 